\title{
Discussion of Dynamic Trace Evidence Used in Driver Identification in Traffic Accidents*
}

\author{
Huawei Xie \\ Department of Forensic Science \\ Fujian Police College \\ Fuzhou, China 350007
}

\author{
Weixian Lin \\ Fujian Shihao Law Firm \\ Fuzhou, China 350002
}

\begin{abstract}
It is very important to identify the driver accurately in the process of solving traffic accident cases. Driver identification becomes the breach to uncover and properly handle the case, especially when the case is of hit-andrun and reported afterwards or in the condition where the testimony to statements "who is the driver" is inconsistent and questionable. In such cases, we should be focused to find the key to trace material evidence, analyze dynamic material evidence of mark, deduce the traffic accident in three dimensional space and temporal evolution of the space, restore the truth, and then comprehensive analysis and identify the real drivers, and lay a foundation for the detection of traffic accidents to safeguard social justice and social stability.
\end{abstract}

Keywords-trace evidence; dynamic evidence; traffic accident; driver; identify

\section{INTRODUCTION}

With the rapid development of the social economy as well as soaring travel and transport needs, road traffic in China has been at increased risk for a long time. Road accidents frequently happened [1], [2], and [3]. The handling of the accident has become the focus of social attention. In particular, the number of casualties and the major traffic accidents with a wide range of impact, the social attention is extremely high. Inspection and identification of road accidents are crucial to find the truths and causes of accidents, and the identification of the trouble-causing drivers becomes the key to dispose of traffic accidents. Whether the driver can correctly identify is not only related to the fair handling of traffic accidents, but also to social justice and social stability. Once there is no clear driver, the handling of the accident is often unable to enter the legal process or even form a wrong case. In this way, the real perpetrators may be allowed to escape due legal sanction. The legitimate rights and interests of the victims and their families are harmed. Justice is compromised. But in reality, on the one hand, the judicial identification of traffic accidents in China is still in the development stage. The inspection and appraisal standards are not matched. Methods and procedures are too simplistic and stylized. The complete and scientific quality assurance system and the quality operation mechanism have not yet been formed. On the other

*Funding: Education Department in Fujian: A (JAT170664) for financial support. hand, traffic accident presents the objective trend of diversity and complexity. In addition to the intentional evasion of the accident perpetrator, it becomes more difficult to identify the driver of the vehicle. It's realistic to strengthen the research on this issue and provide technical theoretical support for judicial handling practice.

\section{A SUMMARY OF DYNAMIC TRACE EVIDENCE}

Dynamic evidence refers to the type of evidence that complies with the law. It passes legal inspection, identification, appraisal, quality certificate and other procedures, and other evidence or the fact of the case, and can have the actual proof of the facts. Dynamic evidence has the following characteristics: First, the dynamic evidence must conform to the legal evidence category. If it doesn't conform to the legal evidence category, then you can't prove it in litigation. Secondly, the dynamic evidence is converted from static evidence. The method of transformation is through legal inspection, identification, identification, quality certificate and other procedures. It makes the static evidence relevant to the facts of the case. In this way, static evidence is turned into dynamic evidence to prove the facts of the case. Third, dynamic evidence can directly prove the facts of the case. It can prove one aspect of a crime [4].

Traffic accident is complicated, various and full of changeability, and there are many factors involved, such as human-beings, vehicles, roads, environments, management and so on[5]. Theoretically speaking, the more evidence of the accident you get, the more accurate the recognition of the driver is. However, a scene of traffic accident is an open environment, and it suffers numerous interferential factors, and often, there are omissions and mistakes in the collection of evidence, which fails to reconstruct accidents scene as a whole and make accurate recognition of drivers. So, inspection and identification officers should spot crucial traces when they inspect the scene or analyze a case through the submitted materials, combine it with other information to make a dynamic trace analysis, analyze the formation mechanism of crucial traces. In this way, the static trace material is converted into dynamic trace material evidence, and we could explore the truth of the accidents.

In conclusion, the dynamic trace evidence is the scientific evidence which could analyze the reasons for the formation of static trace material evidence in the scene of traffic 
accident and deduce the traffic accident in three dimensional space and temporal evolution of the space to restore the truth.

\section{MaIn MATERIAL EVIDENCE OF TRACES USED to IDENTIFY THE DRIVER}

\section{A. The Traces on the Road}

Deformations and traces, inevitably, should be found at the scene of the accident once an accident happened, which could usually mirror the process of the accident. To start with traces on the road, combining with the relevant vehicle traces and material evidence, the motion trails, before accident and after accident, of the causing-trouble vehicle can be compared and analyzed, and then it analyzes the movement process and stress situation of the human body in the vehicle and some other relevant possibilities: which parts may have collision against vehicle components, which channel could the body be thrown out of the car, is there any possibility to find some material evidence of scratches, textiles and blood remained at or around the channel.

\section{B. The Traces on the Vehicle}

The traces outside can show us the ways and times of collisions, and each collision will make those inside to bear the force again; while traces inside can reflect the relationship of the driver's force-bearing against vehicle components. Deforming traces inside are the keys to identify the driver. Analyzing the collided components of the vehicle through the deformed conditions inside can help us to figure out which components may do damage to human body, which has a direct impact on the objective analysis of characteristics and causes of injury formation, the movement forms of human beings during the accident.

\section{The Traces on the Body Surface}

The traces on the body surface refer to the remarks remained on the bodies of the dead or the injury. They are generated during the process of contacting with vehicles, roads and objects in the traffic accident. Generally speaking, the driver's body may have characteristic injury traces, the driver may suffer a crush injury caused by steering wheel, bolstering injury on the arm, impact injury on the lower limbs caused by the instrument board and crush injury caused by left door. Motor-driver may suffer bruise in the perineum and inner thigh, crush injury and impression marks caused by the contacts between figures and motor-barks. Research of the traces on the body surface should base on comprehensive analysis of injured parts, injure characteristics, objects caused the injury and mechanism of injury. Then combine closely with operation conditions of vehicles, force-bearing conditions of the vehicle and the body, traces of the vehicle and textile, attachments and so on. Finally make comprehensive analysis and judgment.

\section{The Traces on the Clothing}

Listing clothing traces as a separate part shows its importance in identifying the driver. Clothing here covers all the wears and ornaments of the body. Components of the vehicle inside sometimes cause traces of tear, slit, fraction and impressing on driver's clothing, in reverse, the comparison between the traces and components of the vehicle could help identify the driver. Scratches on the shoes of motor-driver usually tell you the information of momentary position. Clothing also provides you a clue to identify the driver when you analyzed the monitoring videos.

\section{EXTRACT DYNAMIC TRACE EVIDENCE}

When identified the trouble-causing drivers by dynamic analysis on material evidence, inspection and identification officers should fully understand the situations when the accident occurred, then collect relevant materials provided by case undertakes, such as monitoring video, on-site photos, traffic accident responsibility confirmation, interrogation records, etc. A comprehensive and systematic inspection of traces on the road, on the vehicle, on the body surface and on the clothing should be completed and among which they can find some key static trace evidence. Due to the complicated and numerous factors involved in a traffic accident, every road traffic accident needs a different reference to do a dynamic analysis of material evidence. This paper will analyze and elaborate it with two cases.

\section{A. Focus on Scene Investigation}

The investigation of road traffic accident should be comprehensive and detailed. First, examine the accident scene carefully, conduct an inspection of the involved people, vehicles, roads and surrounding environment, and fully understand the situation of the accident. If the surveyor can't get to the scene of the traffic accident in time, he should collect relevant materials provided by case undertakes, such as monitoring video, on-site photos, traffic accident responsibility confirmation, interrogation records, etc.

\section{B. Dig Deep into the Scene of the Accident and Close Trace Information}

A comprehensive and systematic inspection of traces on the road, on the vehicle, on the body surface and on the clothing should be completed and among which they can find some key static trace evidence. The surveyor should collect evidence that can describe the characteristics and physical characteristics of drivers, and pay attention to dig the trace information that can be determined, and then choose the right breakthrough to maximize the value of trace evidence.

\section{Construct an Evidence Value System that Is Interrelated and Corroborated}

The key static trace material is combined with the knowledge of vehicle tectonics, traffic engineering, traffic accident theory, kinematics, forensic pathology and law, and then deduces the traffic accident in three dimensional space and temporal evolution of the space. In this way, the key static trace material turn into trace dynamic evidence to restore the truth. Finally, the driver is identified based on the dynamic trace physical evidence.

Due to the complicated and numerous factors involved in a traffic accident, every road traffic accident needs a 
different reference to do a dynamic analysis of material evidence.

\section{CONCLUSION}

The development history of road traffic accident technology identification is short. Driver identification is a new problem in accident identification. At present, there is no standardized and complete identification system at home and abroad, and there are no suitable textbooks or tutorials, the professional theoretical system and methods available are limited to the experience and knowledge of the specific experts. Therefore, the standard of judgment varies and the quality of appraisal is uneven. It is urgent to unify the relevant identification standards and standardized procedures. Besides, it cultivates the multi-disciplinary talents who are familiar with the multidisciplinary knowledge of vehicle tectonics, traffic engineering, traffic accident theory, kinematics, forensic medicine and law.

\section{REFERENCES}

[1] Liu M, Chen Y, Lu G. The Analysis of Serious Fatal Road Traffic Accidents in China. 2016;81:02013.

[2] Qiao W, Shang B, editors. Current situation analysis and safety countermeasure study on road traffic accidents in China. International Conference on Remote Sensing, Environment and Transportation Engineering; 2011.

[3] Zhang J, editor Characteristics and Prevention Countermeasures of Extraordinarily Serious Road Accidents in China. The Twelfth COTA International Conference of Transportation Professionals; 2012.

[4] Li Fucheng. A new classification of criminal evidence - Discuss static evidence and dynamic evidence. Chinese journal of criminal law.2013(3):86-96.

[5] Zhang HX. Analysis on Affection and Dissemination of Road Traffic Accidents. Advanced Materials Research. 2012;468-471:200-203. 